\title{
Assessing the Unmanned Aerial Vehicles' Surveillance Problems and Actual Solution Options from the Different Stakeholders' Viewpoint
}

\author{
Bianka Károly, Balázs Sághi \\ ${ }^{1}$ Department of Control for Transportation and Vehicle Systems, Faculty of Transportation Engineering and Vehicle Engineering, \\ Budapest University of Technology and Economics, H-1111 Budapest, Stoczek street 2, Hungary \\ * Corresponding author, e-mail: karoly.bianka@mail.bme.hu
}

Received: 18 January 2019, Accepted: 07 February 2019, Published online: 02 January 2020

\begin{abstract}
The problems related to the integration of the unmanned aerial vehicles into national airspaces is one of the main topics that the scientific researchers are dealing with these days. Despite that, none of them are investigating the UAVs from the surveillance point of view. For that reason, highlighting the problems that have to be dealt with and assessing them through a holistic approach is the aim of the paper. Therefore, the paper takes the different stakeholders view and identify the key factors that have to be taken into account. The authors propose the surveillance technologies that can be considered and evaluated them along the revealed factors through a global view. The results can be applied as a base for the further research in UAVs surveillance domain.
\end{abstract}

\section{Keywords}

UAV surveillance, surveillance-chain, semi-passive surveillance, phased array radar

\section{Introduction}

Over the last couple of years, due to the rapid development of technology and ease of use, airspace users such as unmanned aerial vehicles have spread widely. Devices initially used only for military purposes, nowadays are also commercially available to civilians without the need for any pre-qualification. In subsequent years the unmanned aircraft traffic is expected to grow at an accelerated rate as more and more businesses recognize the potential of their use. (EASA, 2016; Sándor, 2017) This predicted increase may be promoted further by the fact that technological advances are becoming less expensive which allows the price of these devices to decrease.

Unmanned Airborne Vehicles (UAVs), on the basis of the definition of the ICAO Annex 2 (ICAO, 2005) and national laws like the Hungarian legislation, Act XCVII of 1995 on aviation, are considered as aircraft. Currently, the European Aviation Safety Agency (EASA) has defined rules only for UAVs with an operating mass above $150 \mathrm{~kg}$. Below that weight, UAVs are under Member State competence. At present, the Hungarian legal system has not yet established any regulation applicable under the $150 \mathrm{~kg}$ weight limit, but one is currently being developed. This Hungarian case well represents the problem that within
Member states regulations do not apply a consistent approach or even they do not have any adopted rules for light UAVs (EASA, 2017).

With the purpose of the common, harmonized Europewide regulations, on behalf of the European Commission, EASA has started to develop the EU-wide legislative framework for unmanned aircraft with an operating mass under $150 \mathrm{~kg}$.

The priority of the European Commission's aviation policy is to ensure aviation safety with directives and regulations (EESC, 2016). Hence, they have declared the aim of the integration of unmanned aircraft into the existing forms of the Air Traffic Management (ATM) system while maintaining the high level of safety (Van Eekeren et al., 2017). All this must be done in a way, without jeopardizing the manned aviation but yet provides a possibility for further development in the UAVs sector. (EQSC, 2014)

The integration of civilian UAVs into non-segregated airspace (Fig. 1) is precluded by the fact that current air traffic service systems are designed for conventional manned aircraft and those systems are not capable to deal with these new types of air vehicles. 


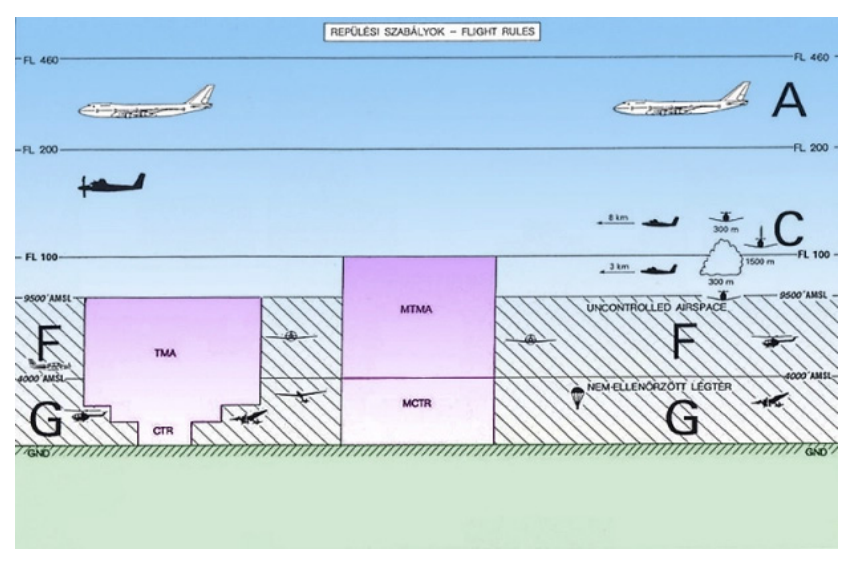

Fig. 1 Air space structure

This is especially true of civil air surveillance systems. The civil surveillance sensors used today are not capable to detect the UAVs which means that they are invisible to the air traffic services.

The aim of this paper is to explain the reasons for this and also to explore potential solutions. For that purpose, an overview of UAVs and current surveillance sensors (Section 2) are provided. In Section 3, the previous findings, the use of alternative methods for UAVs detection are proposed and evaluated in Section 4 using the criteria established by the authors. Finally, in Section 5 the conclusions are drawn and future research directions are summarized.

\section{Methodology}

In this section the UAVs and the current civilian air traffic surveillance are described. Depending on their type and purpose, several terms exist for the aircraft flying without pilot onboard. The most comprehensive term is the Unmanned Airborne System (UAS), which includes both pre-programmed, fully autonomous devices and Remotely Piloted Aircraft Systems (RPAS). Initially, there was a distinction between RPAS and UAS. UAS originally referred to unmanned aircraft which are used for military applications. But they are no longer used only for military purposes but also for commercial and civilian applications. Therefore, the distinction between the two terms is no longer valid and as a result, the term UAS may also apply to unmanned civilian aircraft. Both definitions comprise the entire system required to operate the aircraft shown by Fig. 2 .

Please note abbreviations used on Fig. 2 are:

- the Unmanned Aerial Vehicle (UAV) or Remotely Piloted Aircraft (RPA) itself,

- the control station or the Remote Pilot Station (RPS), from where the aircraft is operated by,

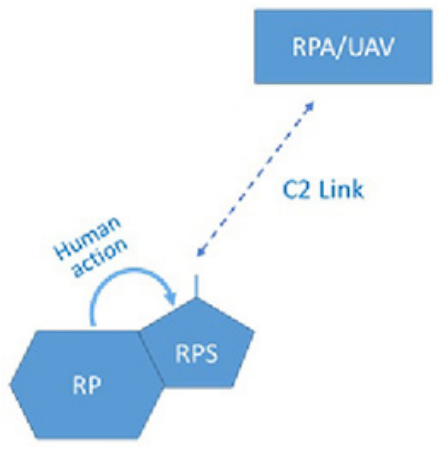

Fig. 2 Elements of an UAS/RPAS

- the human operator called Remote Pilot (RP), and

- the data link, which is called: Command and Control (C2) link.

Another small difference between the RPAS and the UAS terminology is that UAS term includes the possibility that a UAV could be operated in an autonomous mode, without requiring an active $\mathrm{C} 2$ connection with the station. Whereas, a continuous C2 link is required for RPA operation which is unable to fly autonomously. An autonomous UAV is defined as a fully automated pilotless aircraft, which flies a pre-programmed flight path relying on the information collected by its own sensors.

The aforementioned aircraft are known informally as drones in everyday speech. Since we are focusing on the detection of an unmanned aircraft, which is independent of its control, the UAV terminology will be applied in this paper.

\subsection{Description of UAVs}

The markets offer a wide range of UAVs in term of size, performance etc. As a result, they have a wide range of potential applications. As their features differ from traditional aircraft to which the surveillance systems have to adapt, hereinafter we make an overview of these specific features, which is demonstrated by Fig. 2.

Based on the type of aerial platform:

- vertical take-off and landing capable rotary wing aircraft, and

- fixed-wing aircraft for longer flight duration.

Based on their propulsion systems:

- electric propulsion systems,

- fuel propulsion systems.

Almost exclusively, electric UAVs are used for civilian purposes for which the battery ensures the power needed 
for their operation. The operating time of UAVs is determined by the following factors:

- battery capacity,

- UAV weight,

- battery weight,

- payload.

Note, that improvement in the battery's specific energy $(\mathrm{kWh} / \mathrm{kg})$ may result in the further advancement of UAVs.

Type of control:

- remotely controlled,

- fully automated pilotless aircraft, which flies a pre-programmed flight path with the support of their sensors like GPS and

- mixed.

The non-autonomous UAVs typically use the 2.42.5 GHz and 5.725-5.875 GHz for remote control, which frequency bands are not subject to authorization. Autonomous UAVs are controlled by GPS signals or their inertial system manage along their predefined path (Fig. 3).

UAVs exist across a wide range of sizes go from very simple, lightweight drones even to conventional aircraft-sized military purposed systems. In addition to their size, their flight range can be also variable. Along these two flight characteristics, the Unmanned Vehicle Systems International (UVSI) made a classification, which is presented in Table 1.

\subsection{Overview of the current civilian air traffic surveillance}

The identification of the airborne aircraft and knowing their position are the most crucial information for the air navigation service provider (ANSP) to ensure safe separation between aircraft at all time. This information is provided to the air traffic controllers through the air traffic

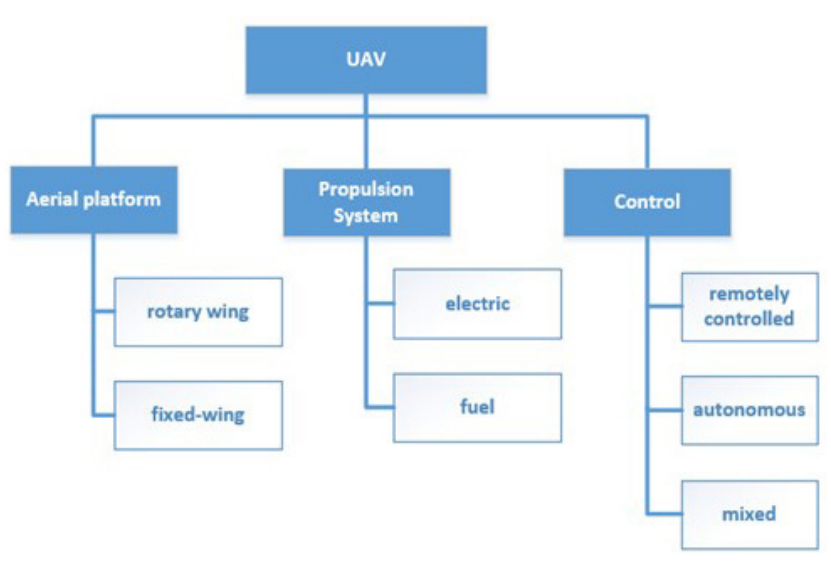

Fig. 3 Classification of the UAVs by their type of their control

surveillance chain via the ATM system. The relationships between the elements forming the surveillance chain are illustrated in Fig. 4. More detailed information is described in Károly et al. (2017).

Since the airborne transponder and the ground surveillance sensor system can be considered as the first elements of the whole surveillance chain, their capabilities fundamentally determine the information that is available. Therefore, in our research presented in this article, we have taken only the transponder and the sensors into account.

Among the civil air surveillance sensors used today, we can distinguish between non-cooperative (passive or primary) and cooperative (also known as active or secondary) systems depending on their working principle.

The passive sensors' position determination does not require any link with the aircraft whereas, for the active sensors' operation on each aircraft, onboard transponder (transmitting responder) is indispensable. Civil air traffic control uses both active and passive surveillance sensors complementing each other. Fig. 5 illustrates their classification.

Table 1 Classification of the UAVs by their physical features

\begin{tabular}{|c|c|c|c|c|c|}
\hline & Long Endurance & Maximum take of weight $(\mathrm{kg})$ & Range (km) & Max Flight Altitude (m) & Endurance (hours) \\
\hline Nano & & $<0.025$ & $<1$ & 100 & $<1$ \\
\hline Micro & & $<5$ & $<10$ & 250 & 1 \\
\hline Mini & & $<30$ & $<10$ & $150-300$ & $<2$ \\
\hline Close Range & & 150 & $10-30$ & 3000 & $2-4$ \\
\hline Short Range & & 200 & $30-70$ & 3000 & $3-6$ \\
\hline Medium Range & & $150-500$ & $70-200$ & $3000-5000$ & $6-10$ \\
\hline Medium Range & $\mathrm{x}$ & $500-1500$ & $>500$ & $5000-8000$ & $10-18$ \\
\hline Low Altitude & $\mathrm{x}$ & $<30$ & $>500$ & 3000 & $>24$ \\
\hline Medium Altitude & $\mathrm{x}$ & $1000-1500$ & $>500$ & 14000 & $24-48$ \\
\hline High Altitude & $\mathrm{x}$ & $2500-12500$ & $>2000$ & $15000-20000$ & $24-48$ \\
\hline
\end{tabular}




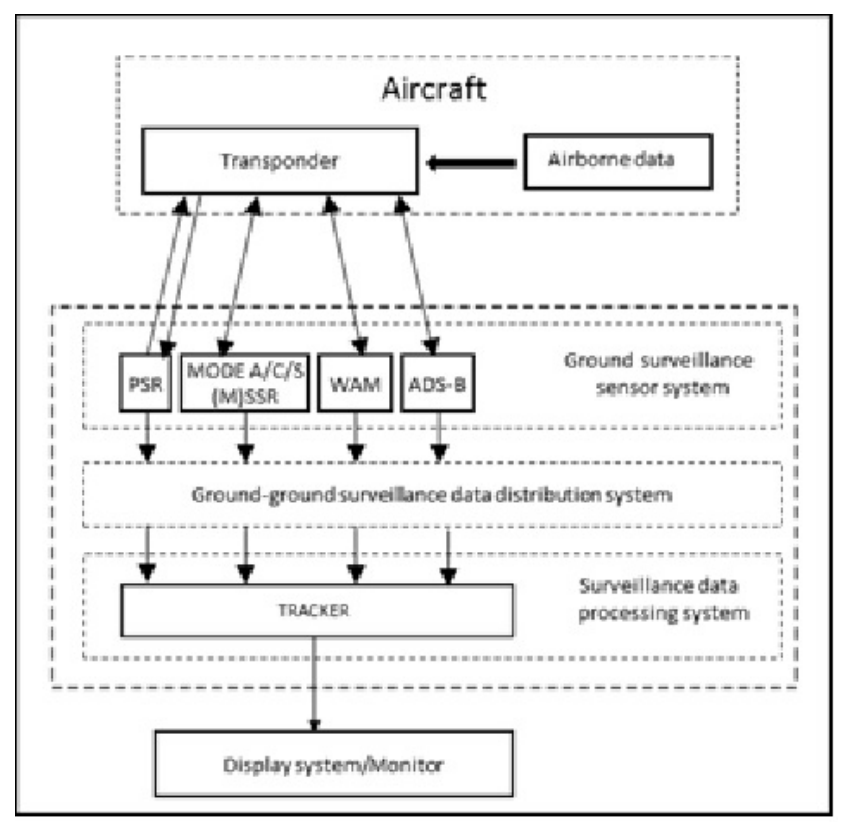

Fig. 4 Elements of the surveillance chain

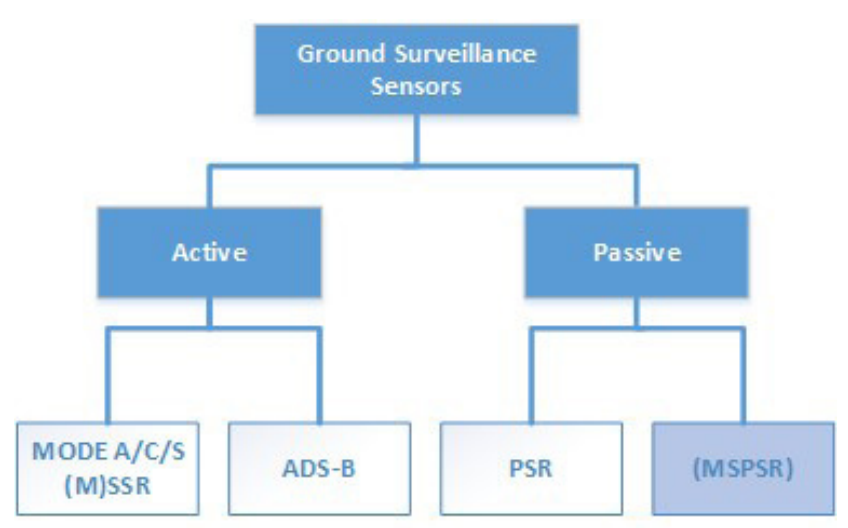

Fig. 5 Surveillance sensors classification

\subsubsection{Passive Surveillance}

Primary Surveillance Radar (PSR) is of paramount importance, as since the 1940s (Bhatta and Geethapriya, 2016), the PSR means the only surveillance form that does not require any cooperation from the airborne aircraft.

Air traffic surveillance radars such TAR, or en-route radars using $\mathrm{S}$ (2.7 to $2.9 \mathrm{GHz}$ ) and $\mathrm{L}$ (1 to $2 \mathrm{GHz}$ ) frequency bands for medium to long range airspace coverage. Their rotating antennas transmit signals with high power, broad bandwidth which are suitable for detecting the conventional, commercial manned aircraft as these signals are reflected from the aircraft surface. Using that reflected radio pulse, it is able to provide basic, but essential range and bearing information about the position of an aircraft in relation to the radar.
PSRs measure this direction, bearing and also the timing and intensity, etc. of the reflected radio waves. The performance is influenced by many factors, such as weather, noise. The main determinant factor one of all is the Radar Cross Section (RCS). Radar cross-section (RCS) is a measure of how detectable an object is by radar (Bothra et al., 2017). An object reflects a limited amount of radar energy back to the source. Many factors have an influence on RCS, such like:

- the material of which the target is made,

- the absolute size of the target.

PSR does not provide with the elevation, identity or detailed position information of the aircraft, meaning it only ensures an elementary picture for air traffic controllers (ATCOs). In order to control the air traffic properly, ATCOs require more advanced data which is provided by the secondary surveillance systems, detailed in the next section.

However, behind PSRs outstanding importance is laying the fact that the detection is guaranteed in case of transponder failure or any unlawful acts.

Beside of PSRs, we have to mention the Multi Static Primary Surveillance Radar (MSPSR) which system is not yet operational, it is only in experimental phase but growing number of research deals with that like NATS Project EVA and Cabalkova et al. (2014). Their results confirmed that MSPSR could serve as an alternative to PSRs.

For the position measurement of an aircraft, MSPSR uses the existing telecommunications and terrestrial broadcasting signal.

The key benefit of MSPSR over conventional PSR is the potential of reduced operating costs, especially where the system exploits non-conventional third-party transmissions such as the TV signals we've been using. More detailed description of this technology is provided in Section 3.2.

\subsubsection{Active Surveillance}

Secondary Surveillance Radar (SSR) is the most widely used active sensor system. As it is a cooperative system, having a transponder onboard is an indispensable condition. Thus, all aircraft operating within controlled or special rules airspace must be equipped with an operational transponder.

The transponder receives and decodes the ground interrogator $1030 \mathrm{MHz}$ pulse signal and responds using a $1090 \mathrm{MHz}$ frequency. The response contains information about the aircraft such as the identification (Mode A) 
and barometric altitude (Mode C). Besides this information, the SSR radar calculates the position of the aircraft. As Károly et al. (2017) reviewed, the latest SSRs systems, called Mode S MSSRs, are able to selectively interrogate a particular aircraft and use the unambiguous identification code and other downloaded aircraft parameters.

More advanced technology and an entirely different position determination are used by the Automatic Dependent Surveillance-Broadcast (ADS-B). Namely, the ADS-B uses the aircraft position data determined by the onboard GPS system, and not by the ground system. This GPS data is automatically transmitted by the transponder, and also contains the position, identification, both barometric and geometric altitudes, velocity, heading and some additional information.

This information is not only received by ground-based sensors, but it is also available to any aircraft equipped with a suitable transponder, for displaying the information onboard. An ADS-B capable transponder is mandatorily to be installed by 2020 for every aircraft above $5700 \mathrm{~kg}$ maximum take-off weight operating in Europe. (Commission Implementing Regulation (EU) 2017/386).

\section{ADS-B has two parts:}

- ADS-B Out and

- ADS-B In.

ADS-B Out is the equipment that transmits the $1090 \mathrm{MHz}$ signals generating the data content mentioned above. ADS-B In is the equipment which is able to receive the $1090 \mathrm{MHz}$ signal from the surrounding area and provide traffic information (situation awareness) to the pilot.

In addition to the direct benefits for pilots, ANSPs could also take advantage of ADS-B. One of the main reason for installing and using ADS-B sensors in comparison to (M)SSRs is that ANSPs could expect medium-, long-term economic benefits as the ground ADS-B sensors mean less hardware and maintenance costs. Moreover, it is also capable of providing surveillance coverage in airspaces that are insufficiently covered by radar.

\section{Results}

Unfortunately, UAVs cannot be detected by either passive or active surveillance sensors described in the previous section. Therefore, in our research, we focused to investigate the reasons behind that, in addition alternative solutions were proposed and assessed.

\subsection{Assessment of the passive UAVs surveillance possibilities}

Since the physical parameters used for the design of civil PSRs used today are targeting the traditional manned aircraft, the UAVs do not mean an effective reflecting surface for them either in size or in materially. In this case, PSRs are not suitable for the UAVs detection. This fact means that UAVs are practically invisible to the air traffic services.

Despite that, air traffic control service is increasingly relying on active surveillance provided data, the importance of UAVs passive surveillance is justified because of their ease of access. As UAVs could mean security threats posed by their unlawful or unintentional usage.

\subsubsection{MSPSR}

Although in today's radar, the transmitter and receiver are collocated, using the same antenna to transmit and receive, if we go back in time to some of the very earliest radar experiments, the transmitter and receiver were geographically separated from each other. These were known as Passive Bistatic Radar, PBR) and Passive Coherent Location (PCL).

The Multi-Static Primary Surveillance Radar is built on this (same) bistatic solution. However, in contrast to PBR and PSR, it does not release any radio signal but exploits third-party transmissions such as telecommunication, like FM, GSM, DTV-B signals. MSPSR uses that these signals are reflected from an aircraft surface.

During its operation, the receiver antenna receives both the direct transmitted and also the reflected signals from an UAV surface. At least the procession of four multi static data pairs like this, results in the target's position.

For the use of air traffic surveillance purposes, it can be said to have a suitable radio tower coverage today, especially at low altitude levels.

The technology is still in the research phase, but there have been good results in several studies targeting UAVs surveillance (Cabalkova et al., 2014; Aldowesh et al., 2015).

Aldowesh et al. (2015) research investigates especially UAV with very low RCS and their detection by MSPSR. The experiment shows that MSPSR systems are able to detect a hardly observable targets. The signal processing chain was able to provide the necessary gain to detect barely observable target at a range of $1 \mathrm{~km}$.

This supports Cabalkova et al. (2014) research that MSPSR can enable not only the manned aircraft without active transponder but also the UAVs detection in the near 
future. The technique may be more suitable for detecting UAVs, since the third-party broadcast signals used, concur with the lower altitude airspace used by the UAVs.

However, the currently available short (about $1 \mathrm{~km}$ ) coverage range requires placing the receiver antennas densely in the area of airspace to be surveyed. This would result in large ground infrastructure investment and thus cost which in the end, have a negative impact on the implementation of the system.

\subsubsection{Phased Array Radar}

Another suitable solution for passive detection of UAVs may be the phase-array antenna system. Unlike conventional radars, it uses a non-rotating antenna, which scans the airspace with a two-dimensional digital beam format.

Like the MSPSR, the concept of the phased array radar it is not new. The first phased array radar was developed at the time of the second world war, but these were really costly. Due to the development of computer technology and digital technology, their price has become significantly more favorable for nowadays.

Thanks to this and other advantageous features, it is an increasingly promising passive surveillance solution. It has also positive judgments due to the lack of moving parts as earlier mentioned, so they are less prone to failure, unlike currently used radars.

However, since this is also largely a short range, it is not suitable for passive surveillance of large-scale airspaces, it is only possible to supervise only highly protected airspace e.g. airfields and shooters.

\subsection{Assessment of the active surveillance possibilities}

To detect UAVs with any cooperative sensor, there is a need to equip them along the lines of the traditional aircraft with transponder. However, due to their size, weight and the level of energy consumption, they are not suitable for placement on UAV.

Alternative beacon is required that are adapted to the UAV's specific properties described in Section 2. Taking these into account, the following criteria should be considered:

- low size,

- low weight,

- low power consumption,

- certification,

- low airborne equipment cost,

- low ground infrastructure cost,
- (partly) compatibility with other airborne equipage from the text.

The equipment power consumption is very important for installations in battery powered UAV aircraft. Since, it has a direct impact on durability and battery size, weight and cost.

\subsubsection{Application of ADS-B}

There are a number of ideas revealed about UAVs detection could been solved ADS-B Out capable transponder which is smaller in size and in power consumption than manned aircraft's version.

A large number of proposals have already been drawn upon equipping UAVs with ADS-B Out transponder which are much smaller in size and in the power consumption than the ones used in manned aircraft just like Pahsa et al. (2011), Guterres et al. (2016).

This would be an appropriate proposal as it would operate on the same $1090 \mathrm{MHz}$ frequency used by a traditional aircraft. Using the same frequency means the system's main advantage and at the same time its drawbacks as well.

The advantage of using $1090 \mathrm{MHz}$ allows the various airspace users to be interoperable in the simplest possible way. As a result, UAVs could not only be displayed for ATCOs, but also for other aircraft, for those who are directly threatened.

The common use of the spectrum is at the same time a disadvantage of using the system. In this regard, Guterres et al. (2016) have conducted a research. In their study, they simulated the anticipated UAVs traffic, which operates on the other ADS-B frequency ( $987 \mathrm{MHz}$ frequency) used in the United States for the lower level operations of the general aviation. Their research revealed that as the number of UAVs in the airspace increases, with the common frequency, the detection probability decreases. Although Guterres et al. (2016) assessed the $987 \mathrm{MHz}$ frequency, a similar result can be expected for the $1090 \mathrm{MHz}$ frequency. Thus assuming that, all anticipated UAVs in the future would provide location information at $1090 \mathrm{MHz}$ in addition to traditional aircraft, there may be a risk of frequency saturation.

The saturation of the frequency channel is less threat for UAVs rather than for the manned aircraft and means aviation safety issue. Since the reliance on the $1090 \mathrm{MHz}$ is so significant, that in case of their saturation and as its consequence, the lack of the secondary surveillance information, the civil air traffic management partly or wholly 
would be impossible in certain regions of Europe. This would mean unforeseeable risks from the aviation safety point of view, as only primary detection data would be available to ATCOs. In today's air traffic volume, the lack of secondary information would induce a very high workload and risk to the ATCOs. Therefore, the protection of the $1090 \mathrm{MHz}$ frequency band is a key aspect.

We, therefore, consider the idea of equipping with an ADS-B Out transponder all the UAVs to be integrated, does not pose an appropriate solution. So, we propose to delineate clearly the UAVs which are most needed to be directly interoperable with manned aircraft and equipping exclusively them with ADS-B Out.

\subsubsection{FLARM}

Being undetectable is not only a problem for the new UAV, but the majority of the sport aircraft and gliders are almost invisible to air traffic control and to each other as well. The reason for the lack of onboard equipment is to be sought in power supply and cost problems as same as in UAVs case.

Recognizing the problem of these aircraft, several alternatives have been developed, which prices are much lower than the normal aircraft's transponder, as for example the so-called FLARM and its advanced version, called PowerFLARM, and OGN tracker onboard beacon. These equipment use the license-free 868.2-868.4 MHz band, which signals can be received and monitored via the ground-based Open Glider Network (OGN).

FLARM and PowerFLARM are small, low-power radio devices. Basically, they are collision warning systems that with a use of their own integrated GPS sensor, can broadcast their own position and velocity vector. At the same time, they also warn of possible short-term conflicts. The more advanced PowerFLARM is capable to process not only the signals on 868.2-868.4 MHz band, but also the Mode A, C, S and ADS-B signals. Therefore, the PowerFLARM enables (one-sided) compatibility with conventional aircraft. Any of the FLARM equipment, even under the current equipage conditions, could be relevant near high sport aircraft and gliders traffic area.

\subsubsection{Mobile network based surveillance}

Since UAV is a new type of the participants in the air traffic, it is also crucial to consider solutions that were not present in the aviation industry before. One of these solutions is mobile network based positioning and tracking, Takács (2008) provides an overview about the variety of this possible method of surveillance.
Based on the mobile network, the cooperative surveillance of the UAVs can be achieved/realized in a simple and cost-effective way. In order to establish this sort of control, it is necessary to install a GSM/LTE modem on the UAVs with, which weight is much more favorable than transponders. The inserted SIM card in the modem allows continuous GSM based positioning and the position information's transmission. Furthermore, thus the vehicle's identification is also solved. The required communication infrastructure for this solution can be provided by telecommunications operators.

The technology's great advantage is that it does not use the air traffic frequencies, but operates on an independent channel, without saturating frequency bands and compromising air traffic safety. However, it is important to emphasize the need for the adequate adaptation of technology for the data provided on the mobile network to be applicable for aviation purposes.

Nevertheless, the mobile network can be useful not only for surveillance purposes but for even the entire UAV traffic management (UTM) can be realized using mobile technology. According to the writer's opinion, it could mean that the GSM/LTE modem will be a basic equipment on certain UAVs in the future.

\subsection{Definition and assessment of the semi-passive surveillance possibilities}

In addition to the categories of active and passive systems presented earlier, we propose to distinguish semi-passive surveillance systems as a separate group. The literature classifies such systems into the passive category, but we do consider essential to emphasize their operational differences to passive devices with the separate grouping (Fig. 6).

Since the operation of semi-passive systems is not based on the transmission of ground infrastructure, but it

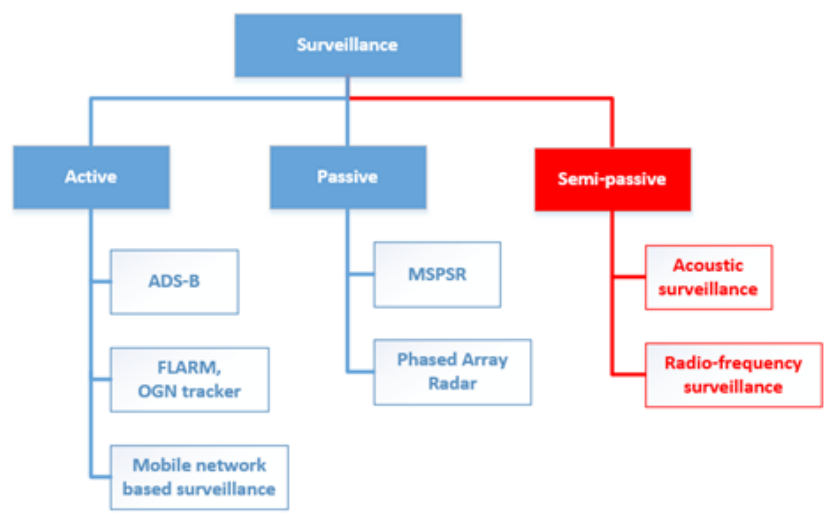

Fig. 6 Extended surveillance sensors classification 
uses the active broadcasts that are involved with the operation of the target, to detect it.

The following solutions are in the semi-passive class:

- acoustic surveillance,

- radio-frequency surveillance.

Military applications have still using them these days, to some extent. In civil applications, they are re-employed as a possible solution for UAVs detection.

\subsubsection{Acoustic surveillance}

Acoustic surveillance detects the presence, the direction, and the frequency of the acoustic vibrations in the free space. The moving elements of the UAVs generate noises and vibrations. Acoustic reconnaissance systems detect these by sensing sound waves generated by UAVs. It has the advantage that it can be used under poor visibility, but the propagation conditions, a noisy environment adversely affect its operation. Furthermore, it can only detect within a short range $(<1 \mathrm{~km})$.

\subsubsection{Radio-frequency surveillance}

For the radio-frequency surveillance, the ground-based system does not transmit any kind of signal that would induce the target to response or that reflects from the targets surface, but the system takes advantage of the signals emitted accompanying to their operation. In the literature, this technology is known as an Emitter Locating System (ELC). Initially, they were only used for military defence purposes. Today, however, with the widespread of UAVs that are hardly producing reflection, it came to the fore in the civil application, too. Its operation is based on the fact that the - not-autonomous- UAVs transmits regularly radio signals such as the $\mathrm{C} 2$ connection related communication - which is essential for non-autonomous UAVs, as C2 provides the connection to/with their ground control.

To this $\mathrm{C} 2$ connection, most commonly the $2.4 \mathrm{GHz}$ and $5.8 \mathrm{GHz}$ frequency signals are used. By monitoring these frequency bands, the majority of the UAVs surveillance can be ensured, taking advantage of the existing active transmission.

However, its applicability is limited: its detection range is greatly restricted and influenced by terrain and local characteristics. Therefore, it can be generally said that the operational area of the system is about $1 \mathrm{~km}$.

\section{Analysis}

As a conclusion of the Section 2, the authors identified the following restrictive factors for UAVs which are essentials to be taken into account in the analysis:

- their size,

- their payload,

- power supply demand.

Based on them Table 2 evaluates the proposed passive UAV surveillance systems in relation to the revealed restrictive UAV factors

Even the increasingly promising passive surveillance solutions such as the MSPSR or the Phased Array Radar cannot cover large areas, therefore we concluded that the passive systems could be only used for highly protected airspace surveillance e.g. airfields.

What the active UAV surveillance possibilities regard, Table 3 evaluates revealed restrictive factors of UAVs.

Although, many research papers suggested the use of the usage of the ADS-B Out equipment on UAVs, our evaluation highlighted the drawbacks that it could cause. We, therefore, consider this idea, to apply to all the UAVs to be integrated, does not pose an appropriate solution. So, we recommend to delineate clearly that the UAVs which are most needed to be directly interoperable with manned aircraft should be equipped with ADS-B Out.

Table 2 Passive surveillance systems from the restrictive factors perspectives

\begin{tabular}{lcccc}
\hline & Range & Price & $\begin{array}{c}\text { Infrastructural } \\
\text { needs }\end{array}$ & $\begin{array}{c}\text { Detection of } \\
\text { autonomous UAV }\end{array}$ \\
\hline $\begin{array}{l}\text { MSPSR } \\
\begin{array}{l}\text { Phased } \\
\text { array radar }\end{array}\end{array}$ & $1 \mathrm{~km}$ & +++ & ++ & yes \\
\hline
\end{tabular}

Table 3 Active surveillance systems from the restrictive factors perspectives

\begin{tabular}{|c|c|c|c|c|c|c|}
\hline & Price & $\begin{array}{c}\text { Size, } \\
\text { Weight }\end{array}$ & Power consumption & Standardization & $\begin{array}{l}\text { Compatibility with } \\
\text { transponder }\end{array}$ & $\begin{array}{c}1090 \mathrm{MHz} \text { frequency } \\
\text { interference }\end{array}$ \\
\hline ADS-B Out & +++ & + & ++ & partly & ++ & yes \\
\hline FLARM & ++ & + & + & partly & + & no \\
\hline OGN & + & + & + & no & - & no \\
\hline GSM & + & + & + & no & - & no \\
\hline
\end{tabular}


As the frequency, affordability, the physical features regards, the LTE $/ 5 \mathrm{G}$ could be one of the best solution for UAVs. This technology could be not only useful for surveillance purposes but also could have a bigger role in the future of unmanned traffic management. Its only drawbacks, that it is not interoperable with the manned aviation transponders.

We concluded a similar result with the proposed new category: semi-passive surveillance solutions that are shown by Table 4 .

It can be said, that the semi-passive solution takes advantage of the unique features of UAVs such as the 2.4 / $5.8 \mathrm{GHz}$ data connection. However, the detection range of these systems makes only feasible to be used as a limited, local solution.

From these results, it appears that the technology already serves with several solutions. Nevertheless, to their application, the lack of standardization means as one of the most significant barrier factors. Therefore, to exploit the potential of the low coast beacons, maturing the applicable standards is required, which providing a clear, identical operating framework for these pieces of equipment.

However, it is important to consider the fact that any alternative transponder placed on the UAVs means additional cost which imposing a barrier to the secondary surveillance of the UAVs. In addition to that, the power supply of the transponder could also mean a challenge, since the onboard equipment would require extra energy capacity.

For smaller UAVs, where the encoder's size, weight, current consumption, and last but not least the cost of the encoder, does not always make it probable that the operators would be willing to equip there UAVs with beacon system.

Especially given the fact that they are obliged to purchase a device that has a negative impact on the operating parameters and performance indicators of their devices, what is more, the functions of those devices are only important for air traffic control.

\section{Conclusion}

The future aviation environment will be extremely challenging from the surveillance infrastructure perspective: it will have to cope with the growth of the UAVs and have to support their safely integration into the current air traffic system.

As the first step of our present research, the problem of the UAVs was highlighted from the ATM and the surveillance perspective. To that for a grounded solution proposal, we consider substantial to provide a complete understanding of the UAVs physical features, which have the main effects on their detectability. The key factors were identified that was essential to the further holistic evaluation.

Then, we presented an overview of the civil air traffic surveillance sensors used today and discussed why they are not capable to detect the UAVs.

Based on the conclusion of the previous chapters, we made the proposals for the use of alternative technical solutions. These solutions were assessed through the revealed key factors.

From our research, it appears that the technology already serves with several solutions. Nevertheless, to their application, the lack of standardization means as one of the most significant barrier factors.

The research we proceed revealed that there is no one perfect solution, which accommodate all stakeholders needs. Therefore, to facilitate all the different needs (e.g. cooperative or non-cooperative), it should be combined the different technologies along an interoperable framework.

The potential of the research field is significant. and a growing issue, therefore, to continue the presented findings: further research can be

- to reveal who are the affected parties concerned with the drone surveillance issues,

- to determine the interoperability requirements for UAV onboard devices,

- to identify the functions associated with airspace surveillance.

Based on these results, an integrated UAS surveillance concept can be formed.

Table 4 Semi-passive surveillance systems from the restrictive factors perspectives

\begin{tabular}{lcccc}
\hline & $\begin{array}{c}\text { Achievable coverage } \\
\text { range }\end{array}$ & Price & Infrastructural needs & $\begin{array}{c}\text { Detection of autonomous } \\
\text { UAV }\end{array}$ \\
\hline Acoustic surveillance & $1 \mathrm{~km}$ & +++ & ++ & yes \\
Radio-frequency surveillance & $1 \mathrm{~km}$ & +++ & ++ & yes \\
\hline
\end{tabular}




\section{References}

Act XCVII of 1995 on aviation Hungarian legislation (1995. évi XCVII. törvény a légiközlekedésröl). (in Hungarian)

Aldowesh, A., Shoaib, M., Jamil, K., Alhumaidi, S., Alam, M. M. (2015) "A passive bistatic radar experiment for very low radar cross-section target detection", In: 2015 IEEE Radar Conference, Johannesburg, South Africa, pp. 406-410.

https://doi.org/10.1109/RadarConf.2015.7411917

Bhatta, N. P., Geethapriya, M. (2016) "RADAR and its Applications", In: International Conference on Novel Issues and Challenges in Science \& Engineering', NICSE'16, Kumarakovil, India, pp. 1-9.

Bothra, B., Rani Panda, A., Pradhan, S., Hussain, A., Dash, D. (2017) "Low RCS Target Detection: A Review", International Journal of Engineering Trends and Technology, 45(4), pp. 137-144. https://doi.org/10.14445/22315381/IJETT-V45P231

Commission Implementing Regulation (EU) 2017/386 of 6 March 2017 amending Implementing Regulation (EU) No 1207/2011 laying down requirements for the performance and the interoperability of surveillance for the single European sky 2017, Official Journal of the European Union, Brussels.

Cabalkova, P., Kubal, D., Pelant, M., Plsek, R., Stejskal, V. (2014) "Aspects of target detection in MSPSR system under clutter conditions", In: 2014 15th International Radar Symposium (IRS), Gdansk, Poland, pp. 1-4.

https://doi.org/10.1109/IRS.2014.6869190

European Economic and Social Committee, EESC (2014) "COM(2014) 207 final "Opinion of the European Economic and Social Committee on the communication from the Commission to the European Parliament and the Council — A new era for aviation - Opening the aviation market to the civil use of remotely piloted aircraft systems in a safe and sustainable manner COM(2014) 207 final", Official Journal of the European Union, The Publications Office of the European Union, Brussels, Belgium.

European Economic and Social Committee, EESC (2016) "Opinion of the European Economic and Social Committee on the Aviation package II, including a'Proposal for a regulation of the European Parliament and of the Council on common rules in the field of civil aviation and establishing a European Union Aviation Safety Agency, and repealing Regulation (EC) No 216/2008 of the European Parliament and of the Council' (COM(2015) 613 final - 2015/0277 (COD)) and a 'Report from the Commission to the European Parliament and the Council - The European Aviation Safety Programme' (COM(2015) 599 final) ", Official Journal of the European Union, The Publications Office of the European Union, Brussels, Belgium.
EASA "Civil drones (Unmanned aircraft)", [online] Available at: https:// www.easa.europa.eu/easa-and-you/civil-drones-rpas [Accessed: 12 November 2017]

European Aviation Safety Agency, EASA (2016) "Study and Recommendations regarding Unmanned Aircraft System GeoLimitations", European Aviation Safety Agency, Cologne, Germany, Rep. TE.GEN.00400-003- [pdf] Available at: https:// www.easa.europa.eu/sites/default/files/dfu/GTF\%20-\%20Report_ Issue2.pdf [Accessed: 17 March 2017]

Guterres, M., Jones, S., Orrell, G., Strain, R. C. (2016) "ADS-B Surveillance in High Density SUAS Applications at Low Altitudes", In: International Symposium on Enhanced Solutions for Aircraft and Vehicle Surveillance Applications, Berlin, Germany. https://doi.org/10.2514/6.2017-1154

ICAO (2005) "ICAO Annex 2, Rules of the Air", International Civil Aviation Organization, Montreal, Canada.

Károly, B., Meyer, D., Sághi B. (2017) "A polgári légtérfelderítő szenzorok fejlesztése" (The developments of the civilian air traffic surveillance sensors), presented at Conference on Innovation and Sustainable Surface Transport, Budapest, Hungary. (in Hungarian)

NATS Project EVA (n.d.) [online] Available at: http://www.nats.aero/ projecteva/ [Accessed: 27 August 2016]

Pahsa, A., Kaya, P., Alat, G., Baykal, B. (2011) "Integrating navigation \& surveillance of Unmanned Air Vehicles into the civilian national airspaces by using ADS-B applications", In: 2011 Integrated Communications, Navigation, and Surveillance Conference Proceedings, Herndon, USA, pp. J7-1-J7-7. https://doi.org/10.1109/ICNSURV.2011.5935334

Sándor, Zs. (2017) "Challenges Caused by the Unmanned Aerial Vehicle in the Air Traffic Management", Periodica Polytechnica Transportation Engineering, 47(2), pp. 96-105. https://doi.org/10.3311/PPtr.11204

Takács, Gy., (2008) "Helymeghatározás mobiltelefonnal és mobil hálózattal" (Positioning with mobile phone and mobile network), Híradástechnika, 68(8), pp. 20-27. (in Hungarian)

Van Eekeren, R., Wright, S., Čokorilo, O. (2017) "A Method of Estimating the Costs of Unexpected Runway Closures Due to Accidents and Incidents", International Journal for Traffic and Transport Engineering, 7(3), pp. 283-297. https://doi.org/10.7708/ijtte.2017.7(3).01 